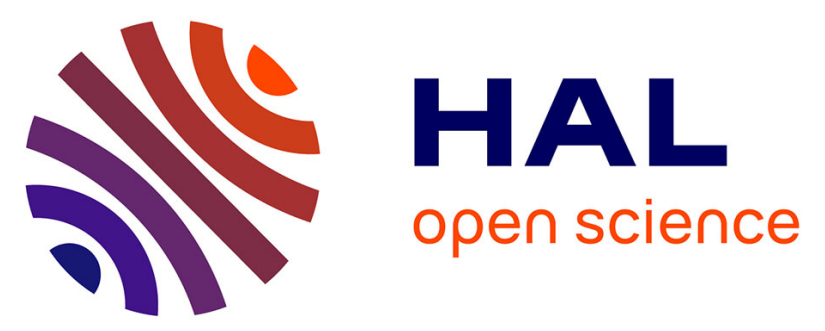

\title{
Abelianity conjecture for special compact Kähler threefolds
}

Frédéric Campana, Benoît Claudon

\section{To cite this version:}

Frédéric Campana, Benoît Claudon. Abelianity conjecture for special compact Kähler threefolds. Proceedings of the Edinburgh Mathematical Society, 2014, 57 (1), pp.55-78. 10.1017/S0013091513000849 . hal-00605338

\section{HAL Id: hal-00605338 \\ https://hal.science/hal-00605338}

Submitted on 1 Jul 2011

HAL is a multi-disciplinary open access archive for the deposit and dissemination of scientific research documents, whether they are published or not. The documents may come from teaching and research institutions in France or abroad, or from public or private research centers.
L'archive ouverte pluridisciplinaire HAL, est destinée au dépôt et à la diffusion de documents scientifiques de niveau recherche, publiés ou non, émanant des établissements d'enseignement et de recherche français ou étrangers, des laboratoires publics ou privés. 


\title{
ABELIANITY CONJECTURE FOR SPECIAL COMPACT KÄHLER THREEFOLDS
}

\author{
FRÉDERIC CAMPANA, BENOÎT CLAUDON
}

Dedicated to $V$. Shokurov.

\begin{abstract}
Using orbifold metrics of the appropriately signed Ricci curvature on orbifolds with negative or numerically trivial canonical bundle and the two-dimensional Log Minimal Model Program, we prove that the fundamental group of special compact Kähler threefolds is almost abelian. This property was conjectured in all dimensions in [Cam04b], and also for orbifolds in [Cam07], where the notion of specialness was introduced. We briefly recall below the definition, basic properties, and the role of special manifolds in classification theory.
\end{abstract}

\section{INTRODUCTION}

We denote here by $X$ an $n$-dimensional compact connected Kähler manifold. The motivations for the following shortest, but non-transparent, definition of specialness will be explained below. See [Cam04b] where this notion was introduced for more details.

Definition 1.1. A compact Kähler manifold $X$ is said to be special if, for any $p>0$, any rank-one coherent subsheaf $\mathcal{L} \subset \Omega_{X}^{p}$ and any positive integer $N$, the natural meromorphic map $\Phi_{N . \mathcal{L}}: X \rightarrow \mathbb{P}\left(V_{N, \mathcal{L}}^{*}\right)$ has an image of dimension at most $(p-1)^{1}$. Here $V_{N, \mathcal{L}}^{*}$ denotes the dual of the complex vector space of sections of $\operatorname{Sym}^{N}\left(\Omega_{X}^{p}\right)$ which take values in $\mathcal{L}^{\otimes N} \subset \operatorname{Sym}{ }^{N}\left(\Omega_{X}^{p}\right)$ at the generic point of $X$.

In particular, $X$ has then no surjective meromorphic map $f: X \rightarrow Y$ onto a manifold $Y$ of general type and dimension $p>0$, since otherwise $\mathcal{L}:=f^{*}\left(K_{Y}\right) \subset$ $\Omega_{X}^{p}$ would contradict the bound $(p-1)$ above. On the non-algebraic side, if $X$ has no map onto a positive dimensional-projective manifold, it is obviously special too. An equivalent geometric definition of specialness of $X$ actually requires that $X$ satisfies the more restrictive condition of having no meromorphic map onto an orbifold of general type (see 3.6 for the precise definition and the relevant notions concerning orbifolds).

Specialness is preserved by bimeromorphic maps and finite élale covers, this last assertion being surprisingly difficult to show. Special manifolds generalise in higher dimensions the rational and elliptic curves, which are obviously exactly the special curves. The next fundamental examples of special manifolds are indeed those which are either rationally connected or whith zero canonical dimension (i.e. with $\kappa=0$, see example 3.2 below). However the class of special manifolds is much larger than the union of these two classes, since one shows by classification that a compact Kähler surface $X$ is special if and only if $\kappa(X) \leq 1$ and $\pi_{1}(X)$ is almost abelian. In particular, ruled elliptic surfaces are special and surfaces with $\kappa(X)=1$ are special

\footnotetext{
Date: July 1, 2011.

${ }^{1}$ By convention, this dimension is $-\infty$ if $V_{N, \mathcal{L}}=\{0\}$ and is always at most equal to $p$ by a classical result of F. Bogomolov.
} 
if and only if they do not map onto any hyperbolic curve after some finite étale cover. No such simple characterisation is true when $n \geq 3$.

The central role of special manifolds in classification theory comes from the fact that, as shown in [Cam04b, 5.8], any compact Kähler manifold is canonically and functorially decomposed by its core fibration $c_{X}: X \rightarrow C(X)$ into its special part (the fibres of $c_{X}$ ) and its general type part (the orbifold base $\left(C(X), \Delta\left(c_{X}\right)\right.$ ), which is its usual base $C(X)$ together with a ramification divisor $\Delta\left(c_{X}\right)$ on $C(X)$ encoding the multiple fibres of $c_{X}$ ).

Special manifolds and general type orbifolds are thus the two antithetical building blocks from which arbitrary compact Kähler manifolds are built in one single step. In contrast to general type manifolds, for which no classification scheme seems to be known or even expected, special manifolds are conjectured to have many fundamental properties in common with rational and elliptic curves.

Conjecturally indeed, an orbifold version of the $C_{n, m}$ conjecture implies that any special manifold is canonically and functorially decomposed, by means of orbifold versions of the rational quotient and of the Iitaka-Moishezon fibration, as a tower of fibrations whose orbifold fibres have either $\kappa=0$, or $\kappa_{+}=-\infty$ (a weak version of rational-connectedness, see [Cam07]). Let us stress that the orbifold considerations are essential here (as in the LMMP, but for different reasons), and that apparently there is no possibility to work in the category of varieties without additional structure. This tower decomposition permits to lift (conditionally) to special manifolds ${ }^{2}$ properties which are expected to be common to manifolds which are either rationally connected or with $\kappa=0$ and naturally leads to the following conjectures:

Conjecture 1.1 ([Cam04b, Cam07]). 1. Abelianity Conjecture: A special compact Kähler manifold has an almost abelian fundamental group (i.e: $\pi_{1}(X)$ has an abelian subgroup of finite index).

2. A compact Kähler manifold (resp. a projective manifold defined over a number field) has an identically vanishing Kobayashi pseudometric (resp. is potentially dense) if and only if it is special (this last statement is inspired by Lang's conjectures).

For example, rationally connected manifold are simply connected [Cam92] and compact Kähler manifolds $X$ with $c_{1}(X)=0$ have an almost abelian fundamental group by [Yau78] and [Bea83].

In this article we prove the Abelianity conjecture above for threefolds (as said above, this is known for surfaces by classification, see Proposition 3.1).

Theorem 1.1. Let $X$ be a compact Kähler threefold. If $X$ is special, its fundamental group is almost abelian.

This immediately implies, among several other things, a precise solution of Shafarevich's conjecture in this case (and for all special manifolds if the Abelianity Conjecture holds):

Corollary 1.1. If $X$ is a special compact Kähler threefold with universal cover $\tilde{X}$, let $X^{\prime}$ be any finite étale cover of $X$ with abelian torsionfree fundamental group and Albanese variety $A l b\left(X^{\prime}\right)$ of dimension $d:=q\left(X^{\prime}\right)$. Then $\tilde{X}=X^{\prime} \times_{A l b\left(X^{\prime}\right)} \widehat{A l b\left(X^{\prime}\right)}$ is holomorphically convex, the universal cover $\widetilde{A l b\left(X^{\prime}\right)}$ of $A l b\left(X^{\prime}\right)$ being Stein, since isomorphic to $\mathbb{C}^{d}$.

\footnotetext{
${ }^{2}$ And even, more naturally, to special orbifolds.
} 


\section{Reduction to the 2-Dimensional orbifold CASE}

We shall prove the theorem in this section only in the cases where no orbifold structure is needed, that is except when either $X$ is projective and $\kappa(X)=2$, or when $a(X)=2$. The treatment of these two residual cases needs the consideration of two-dimensional projective special orbifolds and will be the subject of the subsequent sections.

Because of the lack of a Minimal Model program in the Kähler non-projective case, we need to treat it differently from the projective case. So assume first that $X$ is projective. We work according to the value of $\kappa(X) \leq 2$.

$\boldsymbol{\kappa}(\boldsymbol{X})=-\infty$ : By Miyaoka's theorem, $X$ is uniruled. Let $r_{X}: X \rightarrow R(X)$ be its rational quotient (also known as its MRC fibration). Then $R(X)$ is special too, with $\operatorname{dim}(R(X)) \leq 2$, and $\pi_{1}(X) \simeq \pi_{1}(R(X))$, since the fibres of $r_{X}$ are rationally connected. Since $\pi_{1}(R(X))$ is almost abelian, so is $\pi_{1}(X)$.

$\boldsymbol{\kappa}(\boldsymbol{X})=\mathbf{0}$ : If $c_{1}(X)=0$, the theorem is true, by [Yau78] and [Bea83]. One reduces to this case by the Minimal Model Program and [NS95] (see [Kol95, (4.17.3)]). The details are as follows: there exists a terminal model $X^{\prime}$ birational to $X$ such that $K_{X^{\prime}}$ is torsion, hence trivial after finite étale (in codimension 1) cover. Then $X^{\prime}$ has only $c D V$ singularities. It is thus smoothable in the projective category by [NS95]. The conclusion follows, since $\pi_{1}(X)$ drops by specialisation.

$\boldsymbol{\kappa}(\boldsymbol{X})=1$ : Let $J_{X}: X \rightarrow Y$ be the Moishezon-Iitaka fibration. Because its base is a curve, there exists a finite étale cover of $X$ (still written $X$ ) such that $J_{X}$ has no multiple fibre ${ }^{3}$. Then $\pi_{1}(X)$ is an extension of $\pi_{1}(Y)$ ( $Y$ being a rational or elliptic curve, since special) by a quotient of $\pi_{1}\left(X_{y}\right), X_{y}$ being the generic smooth fibre of $J_{X}$, which is a surface with $\kappa=0$. Because these two groups are almost abelian, $\pi_{1}(X)$ is a priori only polycyclic. The following result however implies the conclusion.

Theorem 2.1 ([Cam01]). Let $f: X \rightarrow Y$ be a fibration without multiple fibres in codimension 1 on $Y$ from a compact Kähler manifold onto a manifold $Y$. Assume that $Y$ and the generic fibre $X_{y}$ of $f$ both have an almost abelian fundamental group. Then $X$ also has an almost abelian fundamental group.

The proof of this theorem rests on two deep results of Hodge theory: Deligne's strictness theorem for morphisms of MHS, and Hain's functorial MHS on the Malčev completion of $\pi_{1}(X)$ when $X$ is compact Kähler.

$\kappa(X)=\mathbf{2}$ : When $X$ is projective, this is the only remaining case. Notice that when $\kappa(X)=1$, we could remove the multiple fibres of $J_{X}$ by making a suitable finite étale cover of $X$ because $Y$ was a curve. When $\kappa(X)=2$ this is no longer possible in general. This is the reason why the notion of orbifold base, which virtually removes the multiple fibres, is introduced in this case, and why the geometry of such orbifolds needs to be developed. But once this is done, and the corresponding properties established, the proof is entirely parallel.

We now deal with the case when $X$ is not projective. We work this time according to the algebraic dimension $a(X) \in\{0,1,2\}$ of $X$.

$\boldsymbol{a}(\boldsymbol{X})=\mathbf{0}:$ The irregularity of $X$ can take the values $q(X)=0,2$ or 3 (since $q(X)=1$ would imply $a(X) \geq 1$ ). The assumption $a(X)=q(X)=0$ leads to the finiteness of $\pi_{1}(X)$ according to [Cam95, Cor. 5.7]. Recall that the Albanese map is connected and surjective when $a(X)=0$. Thus, If $q(X)=3$, then $X$ is birational to its Albanese variety, and $\pi_{1}(X)$ is Abelian. When $q(X)=2$, the

\footnotetext{
${ }^{3}$ Or $Y \cong \mathbb{P}^{1}$ and $J_{X}$ has one or two multiple fibres. This case is easily treated similarly.
} 
Albanese fibration $\alpha_{X}: X \longrightarrow \operatorname{Alb}(X)$ has no multiple fibre in codimension one by [Cam04b, Prop. 5.3]. Moreover, the general fibre of $\alpha_{X}$ is elliptic or rational, by [Uen75, Th. 13.8]. From Theorem 2.1, we conclude that $\pi_{1}(X)$ is almost abelian.

$\boldsymbol{a}(\boldsymbol{X})=1$ : The algebraic reduction $a_{X}: X \rightarrow A(X)$ is then a fibration onto a curve $A(X)$ with general fibre special [Cam04b, Th. 2.39]; the fundamental groups of the base and of the fibre are then almost abelian (see Theorem 3.1). As in the projective case with $\kappa(X)=1$, we may assume that there are no multiple fibres. Then a final application of Theorem 2.1 implies the result.

We are thus left with the following two cases:

(1) $X$ is projective and $\kappa(X)=2$,

(2) $X$ is a compact Kähler threefold with $a(X)=2$.

Let us briefly explain how the conclusion is obtained there. See next sections for the notions which are being used. In both cases we have a fibration (which may be assumed to be neat after suitable modifications of $X$ and $S$ ) $f: X \rightarrow S$ on a smooth projective surface $S$, with generic fibres elliptic curves. This defines a smooth (hence klt) orbifold base $\left(S, \Delta_{f}\right)$. Because $X$ is special, so is $\left(S, \Delta_{f}\right)$. As above, for such a fibration, $\pi_{1}(X)$ is now an extension of $\pi_{1}\left(S, \Delta_{f}\right)$ by $\pi_{1}\left(X_{s}\right)$. We show below:

Theorem 2.2. The fundamental group of a special geometric orbifold $\left(S, \Delta_{S}\right)$ of dimension 2 is almost abelian of even rank at most 4.

The conclusion now follows from the orbifold version of theorem 2.1 above.

Let us stress that our proof of theorem 2.2 depends in an essential way on the fact that integral klt pairs are locally uniformisable by smooth germs of surfaces, because we are using the existence of Ricci-flat orbifold metrics when $c_{1}(S, \Delta)=0$. This is the main reason why we cannot extend the above theorem 2.2 to any higher dimension. Otherwise, using the Abundance Conjecture, it seems that one could derive the Abelianity Conjecture in the projective case for klt orbifolds in any dimension using inductively on the dimension essentially the same arguments as the ones presented below.

\section{SOME BASIC FACTS ON ORBIFOLDS}

3.1. Notion of orbifold. The orbifolds considered here are spaces with local smooth uniformisations under the action of some finite groups. To deal with fixed point in codimension one, the structure is enriched with a $\mathbb{Q}$-divisor of a specific form. The definition below is taken from [GK07].

Definition 3.1. An orbifold is a pair $(X, \Delta)$ where $X$ is a normal variety and $\Delta$ $a \mathbb{Q}$-Weil divisor of the following form:

$$
\Delta=\sum_{i \in I}\left(1-\frac{1}{m_{i}}\right) \Delta_{i},
$$

where the $m_{i} \geq 2$ are integers, and the pair $(X, \Delta)$ is locally uniformizable in the following sense: $X$ is covered by the domains $U$ of finite maps

$$
\varphi: U \longrightarrow X
$$

satisfying the following properties:

(i) $\varphi(U)$ is open in $X$,

(ii) $\varphi: U \longrightarrow \varphi(U)$ is a Galois cover whose branching divisor is exactly $\Delta_{\mid \varphi(U)}$.

The support of $\Delta$ is then: $|\Delta|=\sum_{i} \Delta_{i}$. 


\section{Terminological remark.}

The orbifolds we consider are compatible with all situations in which this term is used: they are particular cases of the ones in [Cam07] and the special manifolds defined below are actually the classical special ones in ibid. They are smooth DeligneMumford stacks too and also klt pairs of the LMMP. Because they are locally smoothly uniformised, we can attach to them fundamental groups and differentialgeometric notions such as metrics and differential forms.

Definition 3.2. The canonical divisor of such a pair is the (Weil) $\mathbb{Q}$-divisor $K_{X}+$ $\Delta$.

Example 3.1. Let $X$ be a smooth variety, and $|\Delta|=\sum_{i} \Delta_{i}$ be a normal crossing divisor; the choice of multiplicities $m_{i} \geq 2$ on each component of the divisor defines a canonical orbifold structure on $(X, \Delta)$. Since $|\Delta|$ is locally given by the equation $z_{1} \cdots z_{k}=0$ in suitable coordinates $\left(z_{1}, \ldots, z_{n}\right)$, the map $\left(z_{1}, \ldots, z_{n}\right) \mapsto$ $\left(z_{1}^{m_{1}}, \ldots, z_{k}^{m_{k}}, z_{k+1}, \ldots, z_{n}\right)$ gives a local uniformization. These orbifolds are said to be smooth and integral in [Cam07].

In particular, an orbifold curve is simply a smooth curve with a finite set of points marked with integral multiplicities at least 2 .

Since we consider only integral and finite multiplicities here, we define:

Definition 3.3. A geometric orbifold is an orbifold $(X, \Delta)$ with $X$ smooth and $|\Delta|$ of normal crossings.

\subsection{Orbifold base of a fibration and special manifolds.}

Definition 3.4 (Def. 1.2, [Cam04b]). Let $f_{0}: X_{0} \longrightarrow Y_{0}$ be a fibration (surjective morphism with connected fibres) between compact complex manifolds. A neat model of $f$ consists in a commutative diagram:

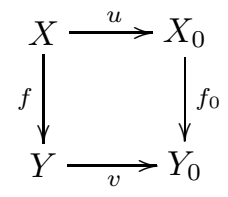

where

1. $X$ and $Y$ are smooth,

2. $u$ and $v$ are bimeromorphic morphisms,

3. the locus of singular fibres of $f$ is a normal crossing divisor of $Y$,

4. every $f$-exceptional divisor is u-exceptional too.

Such models actually exist birationally for any fibration; this can be proven using Hironaka desingularization and Raynaud flattening's theorems [Cam04b, Lemma 1.3]. Notice that any fibration is neat when $Y$ is a curve.

Given a neat fibration $f: X \longrightarrow Y$, we can naturally associate a $\mathbb{Q}$-divisor $\Delta^{*}(f)$ on the base $Y$ of $f$. This divisor will be supported on the singular locus of $f$ and will thus be normal crossings: the pair $\left(Y, \Delta^{*}(f)\right)$ will be a geometric orbifold.

The construction goes as follow (see also [Cam04b, 1.1.4]): if $\Delta_{i}$ is any component of the singular locus of $f$, its pull-back can be written

$$
f^{*}\left(\Delta_{i}\right)=\sum_{j} m_{j} D_{j}+R
$$

where $R$ is $f$-exceptional and $D_{j}$ is mapped surjectively onto $\Delta_{i}$. The multiplicity of $f$ along $\Delta_{i}$ is defined by: $m_{i}=m\left(f, \Delta_{i}\right)=\operatorname{gcd}_{j}\left(m_{j}\right)$. 
Definition 3.5. The pair $\left(Y, \Delta^{*}(f)\right)$, where: $\Delta^{*}(f)=\sum_{i}\left(1-\frac{1}{m_{i}}\right) \Delta_{i}$, is called the orbifold base of the fibration $f_{0}$ (notations as above).

A fibration $f_{0}$ is said to be of general type if the canonical divisor of the orbifold base $\left(Y, \Delta^{*}(f)\right)$ is big: $\kappa\left(Y, K_{Y}+\Delta^{*}(f)\right)=\operatorname{dim}(Y)>0$.

Definition 3.6 ([Cam04b], Def. 2.1). A compact Kähler manifold $X$ is said to be (classically) special if it does not admit any fibration of general type.

Example 3.2. The main examples of special manifolds are given by the following classes [Cam04b, 3.22, 5.1, 2.39]:

$\star$ rationally connected manifolds,

* compact Kähler manifolds $X$ with $\kappa(X)=0$. This is a consequence of the additivity of canonical dimensions in general type fibrations ([Cam04b, Th. 4.2], an orbifold version of Viehweg's theorem).

* The special curves are thus just the rational or elliptic ones.

* compact Kähler manifolds of algebraic dimension zero and more generally fibres of algebraic reductions.

Conjecturally in an orbifold version of Iitaka's $C_{n, m}$-conjecture, special manifolds can be reconstructed as a tower (in a suitable sense) of fibrations with fibres belonging to the classes above. This reduces Abelianity Conjecture 1.1 to the case of orbifolds with either $\kappa=0$ or $\kappa_{+}=-\infty$ (see [Cam07, 13.10]).

In dimension 2 only, we still have a simple topological characterisation of specialness:

Proposition 3.1 (Prop. $3.32,[$ Cam04b]). A compact Kähler surface $X$ is special if and only if: $\kappa(X) \leq 1$ and if $\pi_{1}(X)$ is almost abelian.

We shall need also the notion of orbifold base of a fibration $f: X \rightarrow Y$ also when $X$ is equipped with an orbifold divisor $\Delta_{X}$, at least when $Y$ is a curve and $X$ is a surface (for the general case, see the definition in [Cam07, Def. 4.2]).

Definition 3.7. Let $\left(X, \Delta_{X}\right)$ be a geometric orbifold of dimension 2 and $f: X \longrightarrow$ $C$ a fibration onto a curve. Let us define the multiplicity of a point $y \in C$ (relatively to $f$ and $\left.\Delta_{X}\right)$ by the following formula: $m_{y}\left(f, \Delta_{X}\right):=\operatorname{gcd}_{i}\left(m_{i} \cdot \operatorname{mult}_{\Delta_{X}}\left(F_{i}\right)\right)$, where $f^{*}(y)=\sum_{i} m_{i} F_{i}$. The orbifold base is then the pair $\left(C, \Delta^{*}\left(f, \Delta_{X}\right)\right)$ where:

$$
\Delta^{*}\left(f, \Delta_{X}\right)=\sum_{y}\left(1-\frac{1}{m_{y}\left(f, \Delta_{X}\right)}\right) \cdot\{y\}
$$

We say that $f$ is of general type if: $2 g(C)-2+\operatorname{deg}\left(\Delta^{*}\left(f, \Delta_{X}\right)\right)>0$.

The 2-dimensional orbifold $\left(X, \Delta_{X}\right)$ is then said to be special if $\left(X, \Delta_{X}\right)$ does not admit any general type fibration onto a curve and if $\kappa\left(X, K_{X}+\Delta_{X}\right)<2$.

\section{Fundamental groups AND Fibrations}

4.1. An orbifold exact sequence. In this paragraph, we define fundamental groups of orbifolds and study the morphisms induced at the level of fundamental groups by classical orbifold morphisms.

Definition 4.1. Let $(X, \Delta)$ be an orbifold; its fundamental group $\pi_{1}(X, \Delta)$ is the quotient of the group $\pi_{1}\left(X^{*} \backslash|\Delta|\right)$ by the normal subgroup generated by the loops $\gamma_{j}^{m_{j}}$ where $\gamma_{j}$ is a small loop around the component $\Delta_{j}$ of multiplicity $m_{j}$ (where $X^{*}$ denote the smooth locus of $X$ ).

This definition is derived from the local models. If $X=\mathbb{C}^{n} / G$ where $G$ is a finite subgroup of $\mathrm{GL}_{n}(\mathbb{C})$ and $\Delta$ is the branching divisor of the projection $\pi: \mathbb{C}^{n} \longrightarrow X$, 
we recover the group $G$ as an orbifold fundamental group ${ }^{4}$ :

$$
\pi_{1}(X, \Delta) \simeq G .
$$

Example 4.1. The case of orbifold curves is quite classical. The structure of the fundamental group of such an orbifold curve $\left(C, \Delta=\left(m_{1}, \ldots, m_{n}\right)\right)$ (we just keep in mind the deformation invariant, that is: the multiplicities, not the marked points) is determined by the sign of its canonical bundle:

$$
\operatorname{deg}\left(K_{C}+\Delta\right)=2 g(C)-2+\sum_{j=1}^{n}\left(1-\frac{1}{m_{j}}\right) .
$$

When this quantity is positive (resp. zero, resp. negative), the fundamental group of $(C, \Delta)$ is commensurable to the fundamental group of an hyperbolic curve (resp. commensurable to $\mathbb{Z}^{2}$, resp. finite and explicitely known).

When dealing with fibrations having multiple fibres, we need to consider orbifold fundamental groups. A fibration $f: X \longrightarrow Y$ with general fibre $X_{y}$ gives rise to a natural sequence of fundamental groups:

$$
\pi_{1}\left(X_{y}\right) \stackrel{i_{*}}{\longrightarrow} \pi_{1}(X) \stackrel{f_{*}}{\longrightarrow} \pi_{1}(Y) \longrightarrow 1 .
$$

Although $f_{*}$ is surjective since $f$ has connected fibres, this sequence is in general not exact in the middle. Multiple fibres are responsible for this failure, remedied by the orbifold fundamental group.

We will need the case where $X$ has an orbifold structure and so a slightly more general version. For the definition (of a neat fibration in the orbifold setting), we refer to [Cam07, Def. 4.8].

Proposition 4.1 ([Cam07], Cor. 12.10). Let $f:\left(X, \Delta_{X}\right) \longrightarrow Y$ be a neat fibration. If $X_{y}$ denote a general fibre of $f$ and $\Delta_{y}$ the restriction of the orbifold structure to $X_{y}$, the sequence

$$
\pi_{1}\left(X_{y}, \Delta_{y}\right) \longrightarrow \pi_{1}\left(X, \Delta_{X}\right) \stackrel{f_{*}}{\longrightarrow} \pi_{1}\left(Y, \Delta^{*}\left(f, \Delta_{X}\right)\right) \longrightarrow 1
$$

is exact.

Remark 4.1. We omit the detailled definition of neatness required for the preceding statement because in the sequel we will only use it in the two following quite simple situations:

(a) when $\Delta_{X}$ is empty, this neatness assumption has been already encountered (cf. Definition 3.4). The content of the previous proposition is then that $\pi_{1}(X)$ sits then in the middle of a short exact sequence (with the fundamental group of the orbifold base on the right hand).

(b) when $Y$ is a curve: the fibration $f:\left(X, \Delta_{X}\right) \longrightarrow Y$ is always neat.

4.2. Nilpotency class in fibrations. To complete this study of the behaviour of fundamental groups in fibrations, we prove that nilpotency conditions on the fundamental groups are preserved in fibrations between Kähler orbifolds. This remarkable fact (obviously false even for submersions between complex manifolds as the Iwasawa manifold shows) is a consequence of deep results in Mixed Hodge Theory ${ }^{5}$.

\footnotetext{
${ }^{4}$ The orbifold divisor determines the structure of the smooth Deligne-Mumford stack $\mathcal{X}$ associated with $(X, \Delta)$ and called the root stack by Abramovich-Vistoli.

${ }^{5}$ The key ingredients are: existence of a MHS on the Malčev completion of the fundamental group of a compact Kähler manifold and the strictness of morphisms of MHs.
} 
Theorem 4.1 (Cor. 7.6, [Cam10]). Let $f:\left(X, \Delta_{X}\right) \longrightarrow\left(Y, \Delta_{Y}\right)$ be a neat $f$ bration (see Remark 4.1) between smooth compact Kähler orbifolds. If the groups $\pi_{1}\left(X_{y}, \Delta_{X_{y}}\right)$ and $\pi_{1}\left(Y, \Delta_{Y}\right)$ are almost abelian ${ }^{6}$, then $\pi_{1}\left(X, \Delta_{X}\right)$ is almost abelian as well.

Proof: It is a reduction to the case when $\Delta_{X}=0$. Since $f$ is assumed to be neat, the sequence

$$
\pi_{1}\left(X_{y}, \Delta_{X_{y}}\right) \longrightarrow \pi_{1}\left(X, \Delta_{X}\right) \stackrel{f_{*}}{\longrightarrow} \pi_{1}\left(Y, \Delta_{Y}\right) \longrightarrow 1
$$

is exact. Since $\pi_{1}\left(X_{y}, \Delta_{X_{y}}\right)$ and $\pi_{1}\left(Y, \Delta_{Y}\right)$ are almost abelian groups (of finite type), $G=\pi_{1}\left(X, \Delta_{X}\right)$ is then an almost polycyclic group; in particular, the group $G$ is linear. A famous result of Selberg asserts that $G$ has then a finite index subgroup $G^{\prime} \leq G$ which is torsion free. Consider $X^{\prime}$ the orbifold cover of $\left(X, \Delta_{X}\right)$ associated with $G^{\prime}$; the latter being torsion free, $X^{\prime}$ is a normal variety with no orbifold structure (i.e. $\Delta^{\prime}=0$ ). Since $X$ has only quotient singularities, its fundamental group is isomorphic to the one of $\tilde{X}$, a desingularisation of $X^{\prime}$ [Kol93, Th. 7.5]. To conclude, we consider (a neat model of) the Stein factorization of

$$
\tilde{X} \longrightarrow X^{\prime} \longrightarrow X \longrightarrow Y \text {. }
$$

Indeed, taking further blow-up of $\tilde{X}$, we can complete the picture:

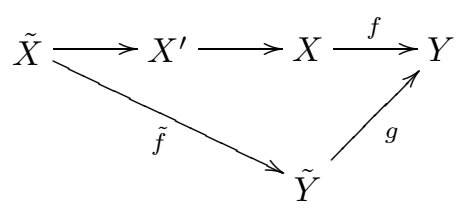

with $\tilde{f}$ a neat fibration and $g$ generically finite. It is then easy to see that we have an exact subsequence (with vertical maps having finite index images):

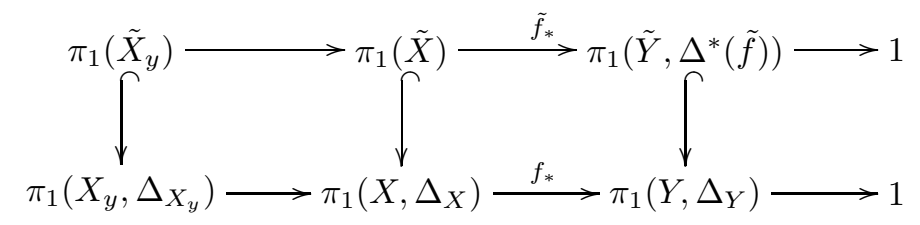

So we are reduced to the same situation with $\Delta_{X}=0$ and we can apply [Cam10, Cor. 7.6].

4.3. Fundamental groups of orbifolds with non-positive canonical bundle. As for of compact Kähler manifolds, when the first Chern class is zero or positive, the now usual differential-geometric methods can then be applied to construct orbifold Kähler metrics with Ricci-curvature of the corresponding sign (using the local smooth uniformisations).

Theorem 4.2. Let $\mathcal{X}=(X, \Delta)$ be an orbifold with $X$ compact Kähler. If the first Chern class of $\mathcal{X}$ is non negative, the group $\pi_{1}(\mathcal{X})$ is almost abelian; more precisely:

$\star$ if $c_{1}\left(K_{X}+\Delta\right)=0$, then $\pi_{1}(X, \Delta)$ is almost abelian of even rank ${ }^{7}$ bounded by $2 \operatorname{dim}(X)$.

$\star$ if $c_{1}\left(K_{X}+\Delta\right)>0$, then $\pi_{1}(X, \Delta)$ is finite.

\footnotetext{
${ }^{6}$ More generally, if these two groups are torsionfree nilpotent of nilpotency class at most $\nu$, then $\pi_{1}\left(X, \Delta_{X}\right)$ is also nilpotent of nilpotency class at most $\nu$.

${ }^{7}$ The rank of an almost abelian group $G$ of finite type is the maximum rank of an abelian subgroup of finite index of $G$.
} 
To begin with, let us recall some basic facts on differential calculus on orbifolds. The smooth functions (differential forms, hermitian metrics,...) on an orbifold $(X, \Delta)$ are the smooth functions on $X^{*} \backslash|\Delta|$ which can be smoothly extended (as the usual objects, after taking inverse images) in local uniformizations. For instance, if $(X, \Delta)$ is a geometric orbifold, a Kähler metric has the following form in coordinates charts adapted to $\Delta$ :

$$
\begin{aligned}
\omega_{\Delta} & =\omega_{e u c l}+\sum_{j=1}^{n} i \partial \bar{\partial}\left|z_{j}\right|^{2 / m_{j}} \\
& =\omega_{e u c l}+\sum_{j=1}^{n} \frac{i d z_{j} \wedge d \overline{z_{j}}}{m_{j}^{2}\left|z_{j}\right|^{2\left(1-1 / m_{j}\right)}} .
\end{aligned}
$$

Local uniformizations can also be used to compute integrals of forms of maximal degree. If $\varphi: U \longrightarrow \varphi(U) \subset X$ is such a local cover and $\alpha$ an orbifold top-form on $X$, define

$$
\int_{\varphi(U)} \alpha=\frac{1}{\operatorname{deg}(\varphi)} \int_{U} \varphi^{*} \alpha
$$

This local computation can then easily be globalized using partitions of unity.

The canonical divisor has been defined in 3.2 as a $\mathbb{Q}$-divisor. It should be noted here that ( $X$ being compact and the uniformizations being finite) some integral multiple of this divisor defines a line bundle on $X$ and it can be used to compute the first Chern class of $X$. As a byproduct, the Ricci form of any orbifold volume form is a $(1,1)$-orbifold form whose cohomology class coincides with $c_{1}(\mathcal{X})$.

As in the manifold case, every invariant form whose cohomology class coincides with $c_{1}(\mathcal{X})$ is the Ricci curvature of an orbifold Kähler metric. This fact has been already noticed several times in the literature (see for instance [Joy00, Cam04a] when $c_{1}(\mathcal{X})=0$, and [DK01] when $c_{1}(\mathcal{X})>0$, and the references therein). For sake of completeness, let us recall the statement.

Theorem 4.3 (Calabi-Yau). Let $\mathcal{X}$ be a Kähler orbifold whose underlying space is compact and let us fix $\omega_{0}$ an orbifold Kähler metric. For any representative (i.e. smooth invariant $(1,1)$ form) $\alpha$ of $c_{1}(\mathcal{X})$, there exists a unique orbifold Kähler metric $\omega$ in the Kähler class $\left[\omega_{0}\right]$ such that: $\operatorname{Ricci}(\omega)=\alpha$.

Proof: We reproduce the arguments given in [Cam04a, Th. 4.1]. This is a simple adaptation of the proof exposed in [Siu87, p. 85-113]. Since Ricci $\left(\omega_{0}\right)$ and $\alpha$ define the same class, the orbifold $\partial \bar{\partial}$-lemma provides us a smooth (orbifold) function $f$ such that $\operatorname{Ricci}\left(\omega_{0}\right)=\alpha+i \partial \bar{\partial} f$. We normalize $f$ (by addition of a suitable constant) in such a way that

$$
\int_{X}\left(e^{f}-1\right) \omega_{0}^{n}=0
$$

The problem is then reduced to solving the Monge-Ampère equation

$$
\text { (MA) } \quad\left(\omega_{0}+i \partial \bar{\partial} \varphi\right)^{n}=e^{f} \omega_{0}^{n} .
$$

To do so, we applied the continuity method as in [Siu87, p. 85-113] and consider the set $T$ of $t \in[0,1]$ for which the equation

$$
(\mathrm{MA})_{t} \quad\left(\omega_{0}+i \partial \bar{\partial} \varphi_{t}\right)^{n}=C_{t} e^{t f} \omega_{0}^{n}
$$

has a solution where

$$
C_{t}=\frac{\int_{X} \omega_{0}^{n}}{\int_{X} e^{t f} \omega_{0}^{n}}
$$


When $t=0, \varphi_{0}=0$ is an obvious solution for $(\mathrm{MA})_{0}$. The set $T$ is open by linearization of the problem and implicit functions theorem (applied in local uniformizations). To see that $T$ is closed we need a priori estimates on the solution of $(\mathrm{MA})_{t}$ which are established using two types of arguments: maximum principle and Nash-Moser iteration (integral inequalities). These can be used in the orbifold setting as well: maximum principle is applied in local uniformizations and integral inequalities are also valid in this formalism (see above).

We proceed as in the manifold case to deduce Theorem 4.2 from Theorem 4.3. The positivity assumptions can be translated in the following way: in the Fano case $\left(c_{1}(\mathcal{X})>0\right)$ there exists a positive $(1,1)$ form $\alpha$ representing $c_{1}(\mathcal{X})$; in the Ricci flat case $\left(c_{1}(\mathcal{X})=0\right)$ we choose $\alpha=0$. We can now apply Theorem 4.3: $\mathcal{X}$ has an orbifold Kähler metric $\omega$ with prescribed Ricci curvature: $\operatorname{Ricci}(\omega)=\alpha$.

In the Fano case, by compactness of $X$, we get: $\operatorname{Ricci}(\omega) \geq \epsilon \omega$, where $\epsilon>0$. The following orbifold version of Myer's theorem applied to the orbifold universal cover $^{8}$ of $\mathcal{X}$ implies the finiteness of the orbifold fundamental group:

Theorem 4.4 ([Bor93]). Let $(\mathcal{Y}, g)$ be a complete Riemannian orbifold (of dimension $n)$. If the Ricci curvature satisfies the inequality

$$
\operatorname{Ricci}(g) \geq(n-1) k
$$

for some $k>0$, the underlying space $Y$ of $\mathcal{Y}$ is then compact with diameter bounded above:

$$
\operatorname{diam}(Y) \leq \frac{\pi}{\sqrt{k}}
$$

When $c_{1}(\mathcal{X})=0$, the following orbifold splitting theorem 4.5 applied also to the orbifold universal cover of $\mathcal{X}$ also implies the claim.

Theorem 4.5 ([BZ94]). Let $(\mathcal{Y}, g)$ be a compact Riemannian orbifold (of dimension $n$ ). If the Ricci curvature is everywhere non negative, the orbifold universal cover $\widetilde{\mathcal{Y}}$ admits a (metric) splitting

$$
\widetilde{\mathcal{Y}} \simeq N \times \mathbb{R}^{m}
$$

where $N$ is a compact orbifold. The orbifold fundamental group of $\mathcal{Y}$ is also an extension of a crystallographic group by a finite group and is in particular almost abelian.

From the arguments given in [Cam04a, 5.4, 6.3] we deduce that the rank of the almost group $\pi_{1}(X, \Delta)$ is even and bouded by $2 \operatorname{dim}(X)$

\section{Minimal Model Program for klt pairs in Dimension 2}

5.1. klt pairs as orbifolds in dimension 2. In this short paragraph, we gather from [KM98] some well known facts on the MMP for log pairs in dimension 2. Starting with a pair $(X, \Delta)$ where $X$ is a smooth surface and $\Delta=\sum_{j} b_{j} \Delta_{j}$ a $\mathbb{Q}$ Weil effective divisor, we can perform a sequence of divisorial contractions which ends with a birational model of the initial pair and whose geometry is simplest, according to the sign of the canonical bundle. In this process, however, $\left(K_{X}+\Delta\right)$ negative curves are being contracted, and the resulting surface is no longer smooth in general. The relevant preserved category of singularities is then described as follows.

Definition 5.1. A pair $(X, \Delta)$ where $X$ is a $\mathbb{Q}$-factorial normal variety is said to have only klt singularities if

$$
\text { (i) } \forall j, 0<b_{j}<1
$$

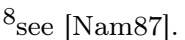


(ii) For any (or equivalently, one) log-resolution $f: Y \longrightarrow X$ of $(X, \Delta)$, in the following equality, we have $a_{i}>-1$, for all $i^{\prime} s$ :

$$
K_{Y}+\tilde{\Delta}=f^{*}\left(K_{X}+\Delta\right)+\sum_{i} a_{i} E_{i},
$$

$\tilde{\Delta}$ being the strict transform of $\Delta$, and $E_{i}$ the exceptional divisors of $f$.

A smooth (integral) orbifold is of course a klt pair. These singularities are preserved in the LMMP:

Theorem 5.1 (Th. 3.47, [KM98]). Let $(X, \Delta)$ be a klt surface. There exists a birational morphism $f: X \longrightarrow S$ such that the resulting pair $\left(S, \Delta_{S}=f_{*}(\Delta)\right)$ is still klt and satisfies (exactly) one of the following properties :

(1) $K_{S}+\Delta_{S}$ is nef,

(2) $S$ admits a fibration $\pi: S \longrightarrow C$ onto a (smooth) curve $C$, the general fibre of $\pi$ being a smooth $K_{S}+\Delta_{S}$-negative rational curve,

(3) $\left(S, \Delta_{S}\right)$ is Del Pezzo $: \rho(S)=1$ and $-\left(K_{S}+\Delta_{S}\right)$ is ample.

When $\Delta=0$, it is well know that klt singularities coincide with quotient singularities in dimension 2 [KM98, Prop. 4.18]. This property still holds for $\Delta \neq 0$ with integral multiplicities (i.e. coefficients $b_{j}$ of the form $1-\frac{1}{m}$ ).

Theorem 5.2. Let $(X, \Delta)$ be a pair where $X$ is a surface and $\Delta$ has integral multiplicities. The following conditions are equivalent near each point of $X$ :

(1) $(X, \Delta)$ is klt,

(2) $(X, \Delta)$ has a finite local fundamental group,

(3) $(X, \Delta)$ is locally presented as a quotient: $\pi: \mathbb{C}^{2} \longrightarrow \mathbb{C}^{2} / G \simeq X$, where $G$ is a finite group acting linearly on $\mathbb{C}^{2}$ and $\Delta$ is the unique $\mathbb{Q}$-divisor on $X$ such that $\pi^{*}\left(K_{X}+\Delta\right)=K_{\mathbb{C}^{2}}$.

This statement is found and used in several places [Kob90, Meg99], but never with a complete accessible proof: the appendix of [Kob90] consists in a list of the possible cases, and refers to the thesis [Nak89] for the proof. For this reason, we give a proof of Theorem 5.2 at the end of the present text ( $c f$. Appendix).

5.2. Fundamental groups and Mori contractions. We now relate the fundamental groups of a geometric orbifold $(X, \Delta)$ of dimension 2 and of its minimal model $\left(S, D=\Delta_{S}=f_{*}(\Delta)\right)$ as in theorem 5.1.

Proposition 5.1. Let $(X, \Delta)$ be a geometric orbifold of dimension 2 and $f$ : $(X, \Delta) \longrightarrow(S, D)$ its minimal model. There is a natural surjective morphism of groups:

$$
f^{\sharp}: \pi_{1}(S, D) \longrightarrow \pi_{1}(X, \Delta) .
$$

In particular, if $\pi_{1}(S, D)$ is almost abelian so is $\pi_{1}(X, \Delta)$.

Proof: Let us call $E$ the union of the curves contracted by $f ; f$ being an isomorphism away from $E$ we have natural maps, $S^{*}$ being the smooth locus of $S$ :

$$
S^{*} \backslash|D| \stackrel{f^{-1}}{\longrightarrow} X \backslash(|\Delta| \cup E) \hookrightarrow X \backslash|\Delta|
$$

(note that $|\Delta|$ and $E$ may have common components). At the level of fundamental groups, we get natural morphisms:

$$
\pi_{1}\left(S^{*} \backslash|D|\right) \stackrel{\sim}{\longrightarrow} \pi_{1}(X \backslash(|\Delta| \cup E)) \rightarrow \pi_{1}(X \backslash|\Delta|) \rightarrow \pi_{1}(X, \Delta) .
$$

To get the morphism $f^{\sharp}$ we need only to remark that the loops around the components of $D$ are sent onto loops around corresponding components in $\Delta$ with the same multiplicities, since $f$ is an isomorphism between $S^{*}$ and $X \backslash E$. 
Example 5.1. In general, the kernel of this morphism can however be very big as shown by the following example.

Let $C$ be an elliptic curve, and let $X$ be the blow-up of $S=C \times C$ at a point $(c, c)$. Let $F \subset S$ the fibre of the first projection through this point and let $G$ be its strict transform in $X$. If $m>1$ is an integer, the minimal model of $\left(X, \Delta=\left(1-\frac{1}{m}\right) G\right)$ is $\left(S, D=\left(1-\frac{1}{m}\right) F\right)$ and the exceptional divisor $E$ of the blow-up is the only $\left(K_{X}+\Delta\right)$-negative curve on $X$. Since the orbifold base of the first projection is $\left(C,\left(1-\frac{1}{m}\right)\{c\}\right)$, the fundamental group of $(S, D)$ has (a finite index subgroup which have) a surjective morphism onto a non-abelian free group. On the other hand the orbifold base of $g:(X, \Delta) \rightarrow C, g$ being the composition of the first projection and the blow-up, is merely $C$ since the fibre over $c$ has $E$ as component and inherits from it the multiplicity 1 . It is then easy to see that $(X, \Delta)$ is special and that its fundamental group is isomorphic to the fundamental group of $X$ and thus abelian.

Remark 5.1. The direction of the arrow $f^{\sharp}$ in proposition 5.1 does not look to be functorial. So we explain its construction differently. The map $f:(X, \Delta) \rightarrow$ $(S, D)=(X, \Delta)_{\min }$ is in general not a 'classical' orbifold morphism (in the sense of [Cam07]) and thus does not induce a functorial morphism of groups. The map $f$ induces an orbifold morphism, and thus a morphism of groups, only when the multiplicities on the exceptional divisors of $f$ are sufficiently divisible (for example by the order of the local fundamental group of $(S, D)$ at the point under consideration). In our example 5.1 above, equipping $E$ with a multiplicity divisible by $m$ is the right condition.

Let thus $\Delta^{+}$be an orbifold divisor on $X$ such that $f_{*}\left(\Delta^{+}\right)=f_{*}(\Delta)=D$, and such that $f:\left(X, \Delta^{+}\right) \rightarrow(S, \Delta)$ and $i d_{X}:\left(X, \Delta^{+}\right) \rightarrow(X, \Delta)$ are orbifold morphisms (this means for $i d_{X}$ that the multiplicity of any component of $\Delta$ divides the corresponding multiplicity in $\Delta^{+}$).

We then get two functorial group morphisms: $f_{+}^{\sharp}: \pi_{1}\left(X, \Delta^{+}\right) \rightarrow \pi_{1}(S, D)$, which is an isomorphism, and $i d_{X}^{\sharp}: \pi_{1}\left(X, \Delta^{+}\right) \rightarrow \pi_{1}(X, \Delta)$, which is surjective (as above). Our initial $f^{\sharp}$ was nothing but $\left(i d_{X}\right)^{\sharp} \circ\left(f_{+}^{\sharp}\right)^{-1}$.

5.3. Abelianity for special klt pairs and proof of the main theorem. To conclude this section, we shall use the preceding proposition to prove Abelianity Conjecture for geometric orbifolds of dimension 2 .

Proof of Theorem 2.2: Let $(S, D)$ the minimal model of the pair $\left(X, \Delta_{X}\right)$. We argue according to the values of $\kappa=\kappa(X, \Delta)=\kappa(S, D) \in\{-\infty, 0,1\}$.

If $\kappa=1$, then $X$ admits a fibration $f$ onto a smooth curve $C$ whose general fiber $F$ satisfies $F \cdot\left(K_{X}+\Delta\right)=0$; the general orbifold fibre $\left(F, \Delta_{F}\right)$ is then orbifoldelliptic and its fundamental group is almost abelian (example 4.1). Adding to $C$ the orbifold divisor $\Delta^{*}=\Delta^{*}\left(f, \Delta_{X}\right)$ of classical multiplicities, we get an exact sequence

$$
\pi_{1}\left(F, \Delta_{F}\right) \longrightarrow \pi_{1}\left(X, \Delta_{X}\right) \longrightarrow \pi_{1}\left(C, \Delta^{*}\right) \longrightarrow 1 .
$$

The orbifold curve $\left(C, \Delta^{*}\right)$ is special since so is $(X, \Delta)$ and Theorem 4.1 shows that $\pi_{1}(X, \Delta)$ is almost abelian.

When $(S, D)$ admits a structure of Mori fibre space over a (special) curve (in particular $\kappa=-\infty$ ), we still have a fibration on $X$ whose fibres are special (in fact rational) and we can proceed as in the lines above.

There are two cases left:

(1) $\kappa(S, D)=0$,

(2) or $(S, D)$ is log Del Pezzo.

In the first case, $K_{S}+D$ being nef it is semi-ample (log abundance for surfaces, see [FM92]) and then torsion. In particular, the orbifold $(S, D)$ is Ricci-flat. In the 
second case, $(S, D)$ is Fano. In both cases, we can apply Theorem 4.2 to conclude that the fundamental group of $(S, D)$ is almost abelian. Finally, Proposition 5.1 can be applied (it is only here we need it) to show that $\pi_{1}\left(X, \Delta_{X}\right)$ is almost abelian as well.

\section{Appendix A. Classification of Klt singularities of Surfaces}

In this appendix we give a proof of the classification of klt singularities for integral pairs (Theorem 5.2). The method is first to treat the singular case (using the method of [KM98, Th. 4.7]) and then to reduce the smooth case to the preceding one using orbifold étale covers. Let then $(X, \Delta), \Delta=\sum_{j}\left(1-\frac{1}{m_{j}}\right) D_{j}$ be a germ of klt pair.

A.1. The singular case. To begin with we quote a useful (negativity) lemma on connected quadratic forms.

Lemma A.1 (Cor. 4.2, [KM98]). Let $E:=\cup_{j} E_{j}$ be a connected exceptional curve on a smooth complex surface. Let $A:=\sum_{j} a_{j} E_{j}$ and $B:=\sum_{j} b_{j} E_{j}$, with $a_{j}, b_{j} \in \mathbb{R}$. Assume that $A \cdot E_{j} \geq B \cdot E_{j}$ for any $j$. Then either $A=B$ or $a_{j}<b_{j}$ for any $j$.

We first consider the case in which the germ $X$ is singular.

Lemma A.2. Assume that $X$ is singular, and that $\Delta \neq 0$. Let $f: X^{\prime} \rightarrow X$ be the minimal resolution of the germ $X, \Delta^{\prime}=\sum_{k}\left(1-\frac{1}{m_{k}}\right) D_{k}^{\prime}$ being the strict transform of $\Delta$ in $X^{\prime}$ and $E=\cup_{j} E_{j}$ the exceptional divisor. The extended dual graph of $f^{*}(\Delta)$ is then one of the following ones below, in which the (non-compact) components of $\Delta^{\prime}$ are indicated by black dots. Moreover, all intersections are transversal and all white dots are smooth rational curves. These are (-2)-curves, except possibly in the first case (see remark $A .1$ below for additional constraints in the first case).

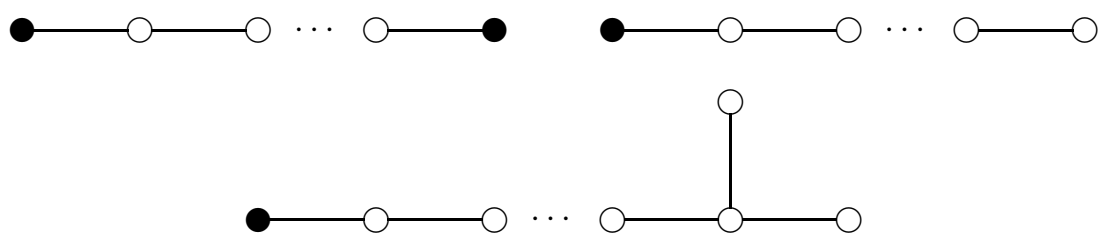

Figure 1. Dual graphs when $\Delta \neq 0$

Proof: We assume the knowledge of the classification of Duval (i.e. canonical) and of klt germs of 2-dimensional singularities, and more precisely the fact that the dual graphs of their minimal resolutions are given either by Dynkin diagrams of (-2)-curves of type $A_{n}, D_{n}, E_{k}$ (for $k=6,7,8$ ) or by Hirzebruch-Jung chains with transversal intersections ([KM98, Th. 4.7], the method of proof we shall now adapt).

From negativity, rational numbers $a_{i}$ are uniquely determined by the numerical equalities:

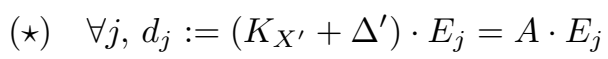

with $A:=\sum_{i} a_{i} E_{i}$. Write also: $e_{i}:=-E_{i}^{2}$.

Assume first that there are (at least) two components $D_{1}$ and $D_{2}$ in $\Delta^{\prime}$, of multiplicities $m_{1}, m_{2}$. Consider a shortest chain $E_{1}, \ldots, E_{n}$ of components of $E$ 
joining them, such that $D_{1}$ (resp. $\left.D_{2}\right)$ meets $E_{1}$ (resp. $E_{n}$ ), but no other $E_{\ell}$. An easy computation gives:

$$
d_{j}=\left\{\begin{array}{l}
\left(e_{1}-2\right)+\left(1-\frac{1}{m_{1}}\right)\left(D_{1} \cdot E_{1}\right) \text { if } j=1, \\
\left(e_{n}-2\right)+\left(1-\frac{1}{m_{n}}\right)\left(D_{2} \cdot E_{n}\right) \text { if } j=n, \\
e_{j}-2 \text { otherwise. }
\end{array}\right.
$$

Consider now the curve $B:=-\beta\left(\sum_{1}^{n} E_{\ell}\right)$, for some $\beta>0$ to be chosen latter. We assume first that $n \geq 2$. The intersection numbers are then given by:

$$
B \cdot E_{j}=\left\{\begin{array}{l}
\beta\left(e_{j}-1\right), j=1, n, \\
\beta\left(e_{j}-2\right), 1<j<n .
\end{array}\right.
$$

We choose now the value of $\beta$ according to the self-intersection of the $E_{j}$ :

(1) if $e_{i} \neq 2$, for some $i \neq 1, n$, we set

$$
\begin{aligned}
\beta & =\inf \left\{1, \frac{d_{j}}{e_{j}-1}, j=1, n\right\} \\
& =\inf \left\{1,1+\frac{\left(1-\frac{1}{m_{1}}\right)\left(E_{1} \cdot D_{1}\right)-1}{e_{1}-1}, 1+\frac{\left(1-\frac{1}{m_{2}}\right)\left(E_{n} \cdot D_{2}\right)-1}{e_{n}-1}\right\}
\end{aligned}
$$

(2) and choose $\beta=\inf \left\{\frac{d_{j}}{e_{j}-1}, j=1, n\right\}$ if $e_{i}=2, \forall i \neq 1, n$.

We thus have $A \cdot E_{j} \geq B \cdot E_{j}$ for any $j$, and equality for some $j$. From Lemma A.1 we get $A=B$ and $a_{j}=-\beta>-1$ since $(X, \Delta)$ is klt. Using ( $\star$ ) it easily implies

$$
\left(1-\frac{1}{m_{1}}\right)\left(D_{1} \cdot E_{1}\right)<1 \text { and }\left(1-\frac{1}{m_{2}}\right)\left(D_{2} \cdot E_{n}\right)<1 \text {. }
$$

Because $\left(1-\frac{1}{m}\right) \geq \frac{1}{2}$ we get $D_{1} \cdot E_{1}=D_{2} \cdot E_{n}=1$ and we are thus in the first case.

Assume now that $n=1$ and notice that then $D_{i}$ meets $E_{1}$ for $i=1,2$. We then have

$$
\left(e_{1}-2\right)+\left(1-\frac{1}{m_{1}}\right)\left(D_{1} \cdot E_{1}\right)+\left(1-\frac{1}{m_{2}}\right)\left(D_{2} \cdot E_{1}\right)=\beta e_{1}^{2}
$$

for some $\beta<1$ (by the klt condition). The solutions are easily determined

(i) either $E_{1} \cdot D_{1}=E_{1} \cdot D_{2}=1$ and $m_{1}, m_{2}$ arbitrary,

(ii) or (up to permutation) $E_{1} \cdot D_{1}=m_{1}=2$ and $E_{1} \cdot D_{2}=1, m_{2}$ arbitrary.

This last case (which is not normal crossings) is excluded by two blow-ups which make the total transform of $\Delta$ a normal crossings divisor. We indeed get the following extended dual graph (in which the $E_{i}^{\prime}, i=1,2,3$ appear as white circles in the order $E_{1}^{\prime}, E_{3}^{\prime}, E_{2}^{\prime}$, while the left (resp. upper) black dot is the strict transform of $D_{1}\left(\right.$ resp. $\left.D_{2}\right)$ :

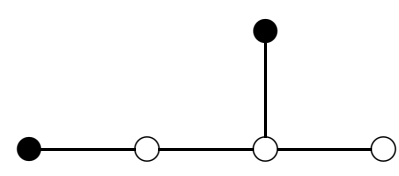

Figure 2. A non-klt case.

We also have: $e_{1}^{\prime}=e_{1}+2, e_{3}^{\prime}=1$ and $e_{2}^{\prime}=2$. A direct resolution of the linear system $(\star)$ shows that this pair is not klt.

Assume next that our dual graph contains a fork (of white vertices). We know that $K_{X^{\prime}} \equiv 0$ in this case $\left(e_{i}=2, \forall i\right)$. Let $D_{1}, E_{1}, \ldots, E_{n-2}, E_{n-1}, E_{n}$ be a shortest subgraph connecting the component $D_{1}$ to the fork $E_{n-2}, E_{n-1}, E_{n}$, with 
end points the last two vertex. The sequence is so labelled that $D_{1}$ meets $E_{1}$ only, and that $E_{1}, \ldots, E_{n-2}$ is a chain. Consider the curve

$$
B:=-\left(1-\frac{1}{m_{1}}\right)\left(D_{1} \cdot E_{1}\right)\left(\sum_{\ell=1}^{n-2} E_{\ell}+\frac{1}{2}\left(E_{n-1}+E_{n}\right)\right) .
$$

A computation similar to the preceding one (but simpler since now $K_{X^{\prime}} \equiv 0$ ) shows that $A \cdot E_{j}=B \cdot E_{j}, \forall j$. Thus $A=B$ and we are in the second case.

The only case left is no fork and only one component in $\Delta^{\prime}$. This is the last diagram (with transversal intersection by the klt and integral conditions, as above) since the degenerate case where $n=2$ in the preceding case would lead to the existence of a fork by connectedness of $E$.

Remark A.1. In the first case, the computation gives additionally: $\left(e_{1}-1\right) m_{1}=$ $\left(e_{n}-1\right) m_{2}$ and also $e_{i}=2, \forall i \neq 1, n$. The arguments of [BHPVdV04, III (5.1)] show that the singularity of $X$ is cyclic of type $A_{N, q}$ with $N=(n-1)\left(e_{1}-1\right)\left(e_{n}-\right.$ $1)+\left(e_{1}-1\right)+\left(e_{r}-1\right)$ and $q=(n-1)\left(e_{1}-1\right)+1$ with $\frac{N}{q}=\left(e_{n}-1\right)+\frac{e_{1}-1}{(n-1)\left(e_{1}-1\right)+1}$.

Lemma A.3. Let $(X, \Delta)$ be a two-dimensional germ of pair as above. This germ is klt if and only if has a finite local fundamental group.

Proof: If $\Delta=0$, it is [KM98, Prop. 4.18]. Otherwise, if the singularity is of type $A_{n}$, an easy computation shows (as in [Lam86, Ch. IV, §13-14]) that the local fundamental group has order $n m_{1} m_{2}$. If the singularity is of type $D_{n}$, we reduce it to the preceding case by a double cover.

To deal with the smooth case, we need preliminary observations (A.4, A.1 and A.6 below) on orbifold étale covers.

A.2. Orbifold étale covers. This first lemma ensure that orbifold étale covers of klt pairs will remain klt.

Lemma A.4 (Prop. 5.20 [KM98]). Let $g:\left(X^{\prime}, \Delta^{\prime}\right) \rightarrow(X, \Delta)$ be a finite map between normal germs of varieties. Assume that $K_{X^{\prime}}+\Delta^{\prime}$ and $K_{X}+\Delta$ are $\mathbb{Q}$ Cartier $^{9}$, and that $K_{X^{\prime}}+\Delta^{\prime}=g^{*}\left(K_{X}+\Delta\right)$. Then $K_{X^{\prime}}+\Delta^{\prime}$ is klt if and only if $K_{X}+\Delta$ is klt.

We come back in dimension 2 with integral pairs.

Definition A.1. We say that a finite map $g:\left(X^{\prime}, \Delta^{\prime}\right) \rightarrow(X, \Delta)$ of degree $d$ is orbifold étale if

(i) the map $g$ ramifies only above the support of $\Delta=\sum_{k}\left(1-\frac{1}{m_{k}}\right) D_{k}$,

(ii) $g$ has order of ramification $r_{k}$ dividing $m_{k}$ along $D_{k}$,

(iii) $\Delta^{\prime}=g^{*}\left(\sum_{k}\left(1-\frac{r_{k}}{m_{k}}\right) D_{k}\right)$.

Remark A.2. In this preceding case the local fundamental group of $\left(X^{\prime}, \Delta^{\prime}\right)$ has index $d$ in the local fundamental group of $(X, \Delta)$. Moreover from the ramification formula we get:

$$
K_{X}^{\prime}+\Delta^{\prime}=g^{*}\left(K_{X}+\sum_{k=1}^{r}\left(1-\frac{1}{r_{k}}\right) D_{k}\right)+\sum_{k=1}^{r}\left(1-\frac{r_{k}}{m_{k}}\right) g^{*}\left(\frac{D_{k}}{r_{k}}\right)=g^{*}\left(K_{X}+\Delta\right)
$$

since $\left(1-\frac{1}{r_{k}}\right)+\left(1-\frac{r_{k}}{m_{k}}\right) \frac{1}{r_{k}}=1-\frac{1}{m_{k}}$. It also follows that $(X, \Delta)$ is klt if and only if so is $\left(X^{\prime}, \Delta^{\prime}\right)$.

We shall apply this remark only when $r_{1}=m_{1}$, and $r_{k}=1$ for $r \geq 2$. In this case $\Delta^{\prime}=g^{*}\left(\sum_{k=2}^{k=r}\left(1-\frac{1}{m_{k}}\right) . D_{k}\right)$ and the component $D_{1}$ 'disappears'.

\footnotetext{
${ }^{9}$ In dimension 2 the $\mathbb{Q}$-Cartier assumption is superfluous.
} 
Example A.1. We now always assume that $X$ is a smooth germ.

1. Assume that $D_{1}$ is a cusp of equation $x^{p}=y^{q}(p, q$ coprime) and of multiplicity $m_{1}$ which we write symbolically as $\Delta_{1}=\left(p, q ; m_{1}\right)$. Then $X^{\prime}$ is the singularity of equation $z^{m_{1}}=y^{q}-x^{p}$. Thus if $(X, \Delta)$ is klt we have $\frac{1}{p}+\frac{1}{q}+\frac{1}{m_{1}}>1$. And so $\left(p, q, m_{1}\right)$ is up to order either $(2,2, m)$ or $(2,3, m), m=3,4,5$. Moreover if the support of $\Delta$ is reducible (if $r \geq 2$ ) then $X^{\prime}$ must be an $A_{n}$ singularity and the support of $\Delta^{\prime}$ must have at most two components. Thus $\left(p, q ; m_{1}\right)$ is, up to order, $(2,2 ; m)$ and $r \leq 3$. If $D_{1}$ is not a priori assumed to be a cusp, but just be given by a parametrisation $x(t)=t^{q}, y(t)=t^{p}$ with $p, q$ coprime, then since the cover of degree $m_{1}$ ramified exactly over $D_{1}$ will be a klt singularity, and so will have the usual normal form in suitable coordinates. We thus see a posteriori that $D_{1}$ was indeed a cusp in suitable coordinates.

2. Assume that the support of $\Delta$ has two smooth components $D_{1}, D_{2}$ tangent at order $p \geq 2$ at the origin. They have (in this order), in suitable coordinates, equations: $y=0$ and $y=x^{p}$. Consider the map: $g: \mathbb{C}^{2} \rightarrow X$ given by: $g(u, v)=$ $(x, y):=\left(u, v^{m_{1}}\right)$. Thus using the above remark A.2, we see that making this (orbifold étale) cover $g$ we are lead to the case (again klt) where

$$
\Delta^{\prime}=g^{*}\left(\sum_{k=2}^{k=r}\left(1-\frac{1}{m_{k}}\right) D_{k}\right),
$$

with $D_{2}^{\prime}$ having equation $v^{m_{1}}=u^{p}$. In this case, letting $d$ be the $g c d$ of $\left(m_{1}, p\right)$, the germ $D_{2}^{\prime}$ splits in $d$ irreducible components which are cusps of type $\left(p^{\prime}, m_{1}^{\prime}\right)$ and multiplicity $m_{2}$ with $p^{\prime}:=\frac{p}{d}, m_{1}^{\prime}:=\frac{m_{1}}{d}$ and are thus smooth if and only if either $p=m_{1}=d$ or $p=d \neq m_{1}$ or $m_{1}=d \neq p$.

A.3. The smooth case. We consider next the remaining case in which the germ $X$ is smooth. Write $\Delta=\sum_{k=1}^{r}\left(1-\frac{1}{m_{k}}\right) D_{k}$. For $k=1 \ldots r$, let $t_{k} \geq 1$ be the multiplicity of the germ $D_{k}$ at the origin. Thus $D_{k}$ is smooth if and only if $t_{k}=1$. We say that an irreducible germ of curve in $X \simeq \mathbb{C}^{2}$ has a $(p, q)$-cusp at the origin if its equation in suitable coordinates is $y^{q}-x^{p}=0$ (with $p$ and $q$ coprime); in this case the multiplicity is $\inf (p, q)$.

Lemma A.5. If $(X, \Delta)$ is klt, the only possibilities for the data $r, t_{k}, m_{k}$ are the following ones.

(1) $\forall k, t_{k}=1$. Then $r \leq 3$. If $r=3$ then $\frac{1}{m_{1}}+\frac{1}{m_{2}}+\frac{1}{m_{3}}>1$ (i.e. either $\left(m_{1}, m_{2}, m_{3}\right)=\left(2,2, m_{3}\right)$ with $m_{3} \geq 2$ arbitrary or $\left(m_{1}, m_{2}, m_{3}\right)=$ $\left(2,3, m_{3}\right)$ with $\left.2 \leq m_{3} \leq 5\right)$.

(2) $t_{k}=2$ for some $k$. Then either $r=1$ and $D_{k}$ has $a(2, q)$-cusp at the origin with $\frac{1}{2}+\frac{1}{q}+\frac{1}{m_{k}}>1$; or $r=2$ (and assume $k=1$ ). There are two subcases:

(a) $D_{1}$ has a $(2, q)$-cusp with $m_{1}=2$ and $D_{2}$ is smooth and has intersection multiplicity 2 with $D_{1} ; m_{2}$ and $q$ (odd) are arbitrary.

(b) $D_{1}$ has a $(2,3)$-cusp with $m_{1}=2, D_{2}$ (smooth) has intersection multiplicity 3 with $D_{1}$ and $m_{2}=2$.

(3) $t_{k}=3$ for some $k$. Then $r=1$ and $D_{1}$ is a $(3,5)$-cusp or $(3,4)$-cusp with $m_{1}=2$.

Proof: Let us make a blow-up $f: X_{1} \rightarrow X$ at the origin with exceptional divisor $E_{1}$ and denote $K_{1}:=K_{X_{1}}$ and $K=K_{X}$. Let $\Delta_{1}$ be the strict transform of $\Delta$ in $X_{1}$. Then $K_{1}+\Delta_{1}=f^{*}(K+\Delta)+c E_{1}$ where $c=1-\sum_{k} t_{k}\left(1-\frac{1}{m_{k}}\right)$. The klt condition implies $c>-1$ that is $\sum_{k} t_{k}\left(1-\frac{1}{m_{k}}\right)<2$. Since $\left(1-\frac{1}{m_{k}}\right) \geq 1$, this implies $\sum_{k} t_{k}<4$. Thus $r \leq 3$ and we get the following list of possible values:

1. if $t_{k} \geq 3$ for some $k$ then $r=1$ and $t_{1}=3$.

2. if $t_{k}=2$ for some $k$ then $r=1,2$ and $\left(t_{1}, t_{2}\right)=(2,1)$ if $r=2$. 
3. if $\forall k, t_{k}=1$ then $r=1,2$ or 3 .

We now examine the distribution of possible multiplicities.

If $t_{1} \geq 2$ and $r=1$ the étale orbifold cover (see A.1 above) of degree $m_{1}$ ramified along the $(p, q)$-cusp $D_{1}$ leads to the singularity $z^{m_{1}}=y^{q}-x^{p}$ with the zero orbifold divisor, which is klt if and only if the claimed inequality $\frac{1}{p}+\frac{1}{q}+\frac{1}{m_{1}}>1$ holds. If $t_{1}=2,3$, we thus get the cases described in cases 2 and 3 of lemma A.5. We are left with the case $t_{1}=2, t_{2}=1, r=2$. Thus $D_{1}$ is a $(2, q)$-cusp of multiplicity $m_{1}$ and $D_{2}$ smooth of multiplicity $m_{2}$ with $q$ odd. We distinguish two cases according to whether the intersection multiplicity of $D_{1}$ and $D_{2}$ is 2 or more.

In the first case, the orbifold étale cover of $(X, \Delta)$ ramified to order $m_{2}$ along $D_{2}$ leads to the orbifold divisor $\Delta^{\prime}$ supported on the locus of equation $x^{2}=y^{m_{2} \cdot q}$ and with multiplicity $m_{1}$. From $\left(X^{\prime}, \Delta^{\prime}\right)$ still being klt we derive $\frac{1}{2}+\frac{1}{q m_{2}}+\frac{1}{m_{1}}>1$ and it yields $m_{1}=2$ since $q m_{2} \geq 6$.

If the intersection multiplicity of $D_{1}$ and $D_{2}$ is 3 or more, the orbifold étale cover of $(X, \Delta)$ ramified to order $m_{2}$ along $D_{2}$ leads to the orbifold divisor $\Delta^{\prime}$ supported on the locus of equation $x^{2 m_{2}}=y^{q}$, and with multiplicity $m_{1}$ (still klt). If $q$ and $m_{2}$ are coprime we get the inequality $\frac{1}{q}+\frac{1}{2 m_{2}}+\frac{1}{m_{1}}>1$ whose the only possible solution is $m_{1}=m_{2}=2$ and $q=3$.

We now show that $q$ and $m_{2}$ are coprime, which will complete the proof. Otherwise let $d>1$ be their $g c d$. Then $\Delta^{\prime}$ consists of $d$ components of multiplicity $m_{1}$ and equations $x^{t}=\varepsilon y^{s}$ with $t=\frac{q}{d}$ and $s=\frac{2 m_{2}}{d}$. These components need to be smooth since $d>1$. Thus either $t=1$ or $s=1$. Since $q$ is odd it is not divisible by $2 m_{2}$ and so $q=d$ divides $m_{2}$. Because $\Delta^{\prime}$ is supported by $d=q \leq 3$ smooth components, we finally get $d=q=3$ components having pairwise tangency of order $\frac{2 m_{2}}{q} \geq 2$ at the origin. This contradicts lemma A.6 below.

Lemma A.6. Let $(X, \Delta)$ be a two-dimensional smooth germ, the support of $\Delta$ consisting of $s \geq 2$ smooth germs having two-by-two contact order $t \geq 1$ at the origin and each of multiplicity $m_{k}(k=1, \ldots, r)$. Then $(X, \Delta)$ is klt if and only if

$$
\sum_{k}\left(1-\frac{1}{m_{k}}\right)<1+\frac{1}{t} \text {. }
$$

The solutions $\left(t, r, m_{1} \leq m_{2} \leq \cdots \leq m_{r}\right)$ are:

$\star t=1, r=2$ and $\left(m_{1}, m_{2}\right)$,

$\star t=1, r=3$ and $\left(2,2, m_{3}\right)$ or $\left(2,3,3 \leq m_{3} \leq 5\right)$,

$\star t=2, r=2$ and $\left(2, m_{2}\right),\left(3, m_{2} \leq 5\right)$,

$\star t=3, r=2$ and $\left(2, m_{2} \leq 5\right)$,

$\star t \geq 4, r=2$ and $(2,2)$.

Proof: We get a log-resolution of this pair in performing $t$ successive suitable point blow-ups with successive exceptional divisors $E_{1}, \ldots, E_{t}$. If $b: X^{\prime} \rightarrow X$ is the resulting composition one checks that in this process

$$
K_{X^{\prime}}=b^{*}\left(K_{X}\right)+\sum_{h=1}^{t} h E_{h}
$$

while

$$
b^{*}(\Delta)=\Delta^{\prime}+\left(\sum_{k}\left(1-\frac{1}{m_{k}}\right)\right)\left(\sum_{h=1}^{t} h E_{h}\right)
$$

where $\Delta^{\prime}$ is the strict transform of $\Delta$. We thus see that the initial pair is klt if and only if the coefficient of $E_{t}$ in $K_{X^{\prime}}+\Delta^{\prime}-b^{*}\left(K_{X}+\Delta\right)$ is strictly greater than -1 , i.e. if

$$
\sum_{k}\left(1-\frac{1}{m_{k}}\right)<1+\frac{1}{t} \text {. }
$$


The solutions are then easily determined.

Proposition A.1. The following list exhaust the classification of integral pairs which are klt with a smooth ambient surface. We use the notation of [Ulu07].
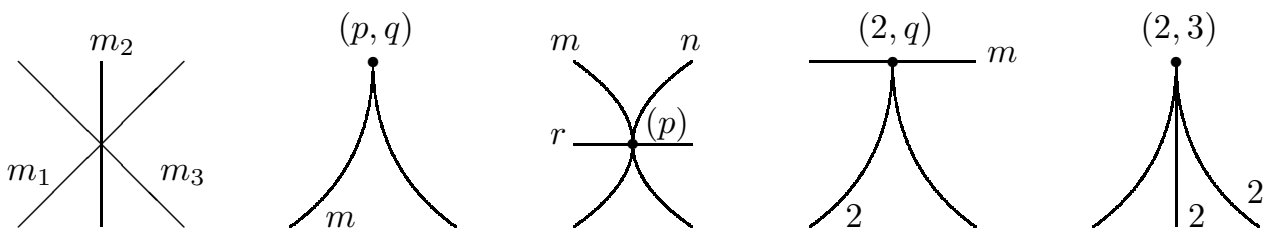

$\sum_{i} \frac{1}{m_{i}}>1$

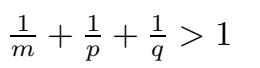

$\frac{1}{m}+\frac{1}{n}+\frac{1}{r p}>1$

$q$ odd

FiguRE 3. Germs of klt pairs with smooth base (and conditions on the coefficients)

Proof: To prove Proposition A.1 we are thus left with the case where $X$ is smooth and $\Delta=\sum_{k=1}^{k=r}\left(1-\frac{1}{m_{k}}\right) D_{k}$, the $D_{k}$ being smooth at the origin. We know that $r \leq 3$. When $r=1$ any $m_{1}$ leads to the klt situation. When $r=2$ the possible situations are described in the lemma A.6. We thus assume that $r=3$ and that not all three components are normal crossings.

We shall then show that (after reordering) $D_{1}$ and $D_{2}$ are tangent to order $t \geq 2$ while $D_{3}$ is transversal to them and that $\frac{1}{m_{1}}+\frac{1}{m_{2}}+\frac{1}{t m_{3}}>1$. So that one has either $m_{1}=m_{2}=2, m_{3}$ and $t$ arbitrary or $2=t=m_{1}=m_{3}$ and $m_{2}=3$.

Assume indeed that $D_{1}$ and $D_{2}$ have contact order $t \geq 2$. We first remark that $D_{3}$ is transversal to them. Assume not, then after possible reordering of the components, we may assume that the order of contact of $D_{3}$ with $D_{1}$ and $D_{2}$ is at least $t$. In this situation, the proof of the above lemma A.6 still applies to show that $\sum_{k=1}^{3}\left(1-\frac{1}{m_{k}}\right)<1+\frac{1}{t} \leq 1+\frac{1}{2}$, which is impossible since $m_{k} \geq 2, \forall k$.

The conclusion now simply follows from considering the orbifold étale cover ramified to order $m_{3}$ along $D_{3}$, which replaces $\Delta$ by $\Delta^{\prime}$, supported by two smooth components of multiplicities $m_{1}$ and $m_{2}$ and having contact order $t^{\prime}=t m_{3}$. This implies by lemma A.6 that $\frac{1}{m_{1}}+\frac{1}{m_{2}}+\frac{1}{t m_{3}}>1$ since the inequality $\sum_{k}\left(1-\frac{1}{m_{k}}\right)<1+\frac{1}{t^{\prime}}$ is equivalent to $\sum_{k} \frac{1}{m_{k}}+\frac{1}{t^{\prime}}>1$ when $r=2$. The solutions are the classical ones.

\section{A.4. The local fundamental groups.}

Proposition A.2. Let $(X, \Delta)$ be a two-dimensional germ of integral pair. If this germ is klt it has a finite local fundamental group.

Proof: If $X$ is singular this is lemma A.3. When $X$ is smooth, this is a direct consequence of the construction of suitable orbifold étale covers as explained above.

\section{REFERENCES}

[Bea83] A. Beauville, Variétés Kähleriennes dont la première classe de Chern est nulle, J. Differential Geom. 18 (1983), no. 4, 755-782 (1984)

[BHPVdV04] Wolf P. Barth, Klaus Hulek, Chris A. M. Peters, and Antonius Van de Ven, Compact complex surfaces, second ed., Ergebnisse der Mathematik und ihrer Grenzgebiete. 3. Folge. A Series of Modern Surveys in Mathematics [Results in Mathematics and Related Areas. 3rd Series. A Series of Modern Surveys in Mathematics], vol. 4, Springer-Verlag, Berlin, 2004.

[Bor93] Joseph E. Borzellino, Orbifolds of maximal diameter, Indiana Univ. Math. J. 42 (1993), no. 1, 37-53. 
[BZ94] Joseph E. Borzellino and Shun-Hui Zhu, The splitting theorem for orbifolds, Illinois J. Math. 38 (1994), no. 4, 679-691.

[Cam92] F. Campana, Connexité rationnelle des variétés de Fano, Ann. Sci. École Norm. Sup. (4) 25 (1992), no. 5, 539-545.

[Cam95] _ Fundamental group and positivity of cotangent bundles of compact Kähler manifolds, J. Algebraic Geom. 4 (1995), no. 3, 487-502.

[Cam01] Ensembles de Green-Lazarsfeld et quotients résolubles des groupes de Kähler, J. Algebraic Geom. 10 (2001), no. 4, 599-622.

[Cam04a] Orbifoldes à première classe de Chern nulle, The Fano Conference, Univ. Torino, Turin, 2004, pp. 339-351.

[Cam04b] Orbifolds, special varieties and classification theory, Ann. Inst. Fourier (Grenoble) 54 (2004), no. 3, 499-630.

[Cam07] Orbifoldes spéciales et classifications biméromorphes des variétés kählériennes compactes, preprint arXiv:0705.0737. To appear in Journal de Mathématiques de Jussieu, 2007.

[Cam10] _ Quotients résolubles ou nilpotents des groupes de Kähler orbifolde, Man. Math. 135 (2010), no. 1-2, 117-150.

[DK01] Jean-Pierre Demailly and János Kollár, Semi-continuity of complex singularity exponents and Kähler-Einstein metrics on Fano orbifolds, Ann. Sci. École Norm. Sup. (4) 34 (2001), no. 4, 525-556.

[FM92] L. Fong and J. McKernan, Log abundance for surfaces, Flips and Abundance for Algebraic Threefolds (J. Kollar, ed.), Astérisque, no. 211, 1992, pp. 127-137.

[GK07] Alessandro Ghigi and János Kollár, Kähler-Einstein metrics on orbifolds and Einstein metrics on spheres, Comment. Math. Helv. 82 (2007), no. 4, 877-902.

[Joy00] Dominic D. Joyce, Compact manifolds with special holonomy, Oxford Mathematical Monographs, Oxford University Press, Oxford, 2000.

[KM98] János Kollár and Shigefumi Mori, Birational geometry of algebraic varieties, Cambridge Tracts in Mathematics, vol. 134, Cambridge University Press, Cambridge, 1998, With the collaboration of C. H. Clemens and A. Corti, Translated from the 1998 Japanese original.

[Kob90] Ryoichi Kobayashi, Uniformization of complex surfaces, Kähler metric and moduli spaces, Adv. Stud. Pure Math., vol. 18, Academic Press, Boston, MA, 1990, pp. 313394.

[Kol93] J. Kollár, Shafarevich maps and plurigenera of algebraic varieties, Invent. Math. 113 (1993), no. 1, 177-215.

[Kol95] János Kollár, Shafarevich maps and automorphic forms, M. B. Porter Lectures, Princeton University Press, Princeton, NJ, 1995.

[Lam86] Klaus Lamotke, Regular solids and isolated singularities, Advanced Lectures in Mathematics, Friedr. Vieweg \& Sohn, Braunschweig, 1986.

[Meg99] G. Megyesi, Generalisation of the Bogomolov-Miyaoka-Yau inequality to singular surfaces, Proc. London Math. Soc. (3) 78 (1999), no. 2, 241-282.

[Nak89] S. Nakamura, Classification and uniformisation of log-canonical singularities in the presence of branch loci (in Japanese), Ph.D. thesis, Saitama University, Japan, 1989.

[Nam87] M. Namba, Branched coverings and algebraic functions, Pitman Research Notes in Mathematics Series, vol. 161, Longman Scientific \& Technical, Harlow, 1987.

[NS95] Yoshinori Namikawa and J. H. M. Steenbrink, Global smoothing of Calabi-Yau threefolds, Invent. Math. 122 (1995), no. 2, 403-419.

[Siu87] Yum Tong Siu, Lectures on Hermitian-Einstein metrics for stable bundles and Kähler-Einstein metrics, DMV Seminar, vol. 8, Birkhäuser Verlag, Basel, 1987.

[Uen75] K. Ueno, Classification theory of algebraic varieties and compact complex spaces, Springer-Verlag, Berlin, 1975, Notes written in collaboration with P. Cherenack, Lecture Notes in Mathematics, Vol. 439.

[Ulu07] A. Muhammed Uludă̆, Orbifolds and their uniformization, Arithmetic and geometry around hypergeometric functions, Progr. Math., vol. 260, Birkhäuser, Basel, 2007, pp. 373-406.

[Yau78] Shing Tung Yau, On the Ricci curvature of a compact Kähler manifold and the complex Monge-Ampère equation. I, Comm. Pure Appl. Math. 31 (1978), no. 3, 339-411.

Fréderic Campana, Benoît Claudon, Institut Élie Cartan Nancy, Université Henri

Poincaré Nancy 1, B.P. 70239, 54506 Vandoeuvre-lès-Nancy Cedex, France

E-mail address: Frederic.Campana@iecn.u-nancy.fr, Benoit.Claudon@iecn.u-nancy.fr 